\title{
AN ALGORITHMIC SIGN-REVERSING INVOLUTION FOR SPECIAL RIM-HOOK TABLEAUX
}

\author{
BRUCE E. SAGAN AND JAEJIN LEE
}

\begin{abstract}
Eğecioğlu and Remmel [2] gave an interpretation for the entries of the inverse Kostka matrix $K^{-1}$ in terms of special rim-hook tableaux. They were able to use this interpretation to give a combinatorial proof that $K K^{-1}=I$ but were unable to do the same for the equation $K^{-1} K=I$. We define an algorithmic signreversing involution on rooted special rim-hook tableaux which can be used to prove that the last column of this second product is correct. In addition, following a suggestion of Chow [1] we combine our involution with a result of Gasharov [5] to give a combinatorial proof of a special case of the $(\mathbf{3}+\mathbf{1})$-free Conjecture of Stanley and Stembridge [14].
\end{abstract}

\section{INTRODUCTION}

We first recall some definitions from the theory of Young tableaux. Further exposition can be found in the texts of Fulton [3], Macdonald [9], Sagan [10], and Stanley [13].

Let $\lambda=\left(\lambda_{1}, \lambda_{2}, \ldots, \lambda_{l}\right)$ be a partition of the nonnegative integer $n$, denoted $\lambda \vdash n$, so $\lambda$ is a weakly decreasing sequence of positive integers summing to $n$. We will also let $\lambda$ stand for the Ferrers diagram of $\lambda$ written in English notation with $\lambda_{i}$ nodes or cells in the $i$ th row from the top. In addition, we will use the notation $\lambda=\left(1^{m_{1}}, 2^{m_{2}}, \ldots, n^{m_{n}}\right)$ which means that the integer $j$ appears $m_{j}$ times in $\lambda$. The set of all partitions of all $n \geq 0$ will be denoted Par.

Given $\lambda, \mu \vdash n$, the corresponding Kostka number $K_{\lambda, \mu}$ is the number of semistandard Young tableaux $T$ of $\operatorname{shape} \operatorname{sh}(T)=\lambda$ and content $c(T)=\mu$, i.e., the number of fillings of the diagram $\lambda$ with integers such that rows weakly increase, columns strictly increase, and for all $k$ the integer $k$ occurs $\mu_{k}$ times. For fixed $n$, we collect these numbers into the Kostka matrix $K=\left(K_{\lambda, \mu}\right)$. We will use the reverse lexicographic order on partitions so that $K$ becomes upper unitriangular.

Eğecioğlu and Remmel [2] gave a combinatorial interpretation for the entries of the inverse Kostka matrix $K^{-1}$ as follows. A rim hook, $H$, is a skew diagram (the settheoretic difference of two ordinary diagrams) which is connected and contains no $2 \times 2$ square of cells. The size of $H$ is the number of cells it contains. A rim-hook tableau

Date: June 14, 2004.

1991 Mathematics Subject Classification. Primary 05E10; Secondary 05A17, 05E05, 06A11.

Key words and phrases. inverse Kostka matrix, algorithmic sign-reversing involution, special rimhook tableau, $(\mathbf{3}+\mathbf{1})$-free Conjecture . 
of shape $\lambda$ is a partition of the diagram of $\lambda$ into rim hooks. This tableau $S$ is special if each of the rim hooks contains a cell from the first column of $\lambda$. The type of $S$ is $t(S)=\left(1^{m_{1}}, 2^{m_{2}}, \ldots, n^{m_{n}}\right)$ where $m_{k}$ is the number of rim hooks in $S$ of size $k$. Using nodes for the Ferrers diagram and connecting them if they are adjacent in the same rim hook, the following diagram illustrates a special rim-hook tableau $S$ with $\operatorname{sh}(S)=\left(1^{2}, 2^{2}, 3\right)$ and $t(S)=\left(1,4^{2}\right)$.

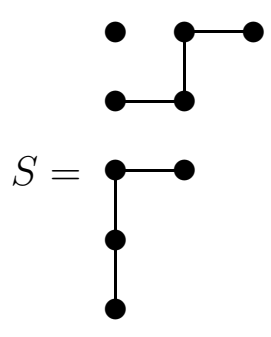

The leg length of rim hook $H, l(H)$, is the number of vertical edges in $H$ when viewed as in the diagram above. We now define the sign of a rim hook $H$ and of a rim-hook tableau $S$ to be

$$
\epsilon(H)=(-1)^{l(H)} \text { and } \quad \epsilon(S)=\prod_{H \in S} \epsilon(H),
$$

respectively. The previous rim-hook tableau has sign

$$
\epsilon(S)=(-1)^{0} \cdot(-1)^{1} \cdot(-1)^{2}=-1 .
$$

We can now state Eğecioğlu and Remmel's interpretation.

Theorem 1.1 (Eğecioğlu and Remmel). The entries of the inverse Kostka matrix are given by

$$
K_{\mu, \lambda}^{-1}=\sum_{S} \epsilon(S)
$$

where the sum is over all special rim hook tableaux $S$ with $\operatorname{sh}(S)=\lambda$ and $t(S)=\mu$.

In [2] they used the combinatorial interpretation in this theorem to give a proof of the fact that $K K^{-1}=I$ using a sign-reversing involution, but were not able to do the same thing for the identity $K^{-1} K=I$. In the next section, we will give an algorithmic sign-reversing involution which will prove that the last column of the second product is correct. Parts of this procedure are reminiscent of the lattice path involution of Lindström [8] and Gessel-Viennot [6, 7] as well as the rim-hook Robinson-Schensted algorithm of White [16] and Stanton-White [15]. In section 3 we follow a suggestion of Chow [1] and combine our involution with a result of Gasharov [5] to prove a special case of the $(\mathbf{3}+\mathbf{1})$-free Conjecture of Stanley and Stembridge [14]. Finally, we end with a discussion of further work which needs to be done. 


\section{The BAsic InVOLUtion}

First note that by Theorem 1.1, $K_{\left(1^{n}\right), \lambda}^{-1}$ is just the number of special rim-hook tableaux of shape $\lambda$ where all hooks have size one (since such tableaux have sign +1 ). But since they must also contain a cell of the first column, such a tableau exists precisely when $\lambda=\left(1^{n}\right)$ and in that case the tableau is unique. Since the definition of the Kostka matrix immediately gives $K_{\left(1^{n}\right),\left(1^{n}\right)}=1$, we have that the inner product of the last row of $K^{-1}$ and the last column of $K$ is 1 as desired. So for the rest of this section we will assume that the type of our special rim-hook tableau is $\mu \neq\left(1^{n}\right)$.

We wish to show that the inner product of the $\mu$ th row of $K^{-1}$ with column $\left(1^{n}\right)$ of $K$ is 0 . It follows from Eğecioğlu and Remmel's Theorem that we need to prove

$$
\sum_{(S, T)} \epsilon(S)=0
$$

the sum being over all pairs $(S, T)$ where $S$ is a special rim-hook tableau with $t(S)=\mu$, and $T$ is a standard Young tableau (that is, $c(T)=\left(1^{n}\right)$ ) of the same shape as $S$. We will prove this identity by exhibiting a sign-reversing involution $I$ on such pairs $(S, T)$.

Suppose first that the cell of $n$ in $T$ corresponds to a hook of size one in $S$. Then since $S$ is special, this cell is at the end of the first column. In this case, remove that cell from both $S$ and $T$ to form $\bar{S}$ and $\bar{T}$ respectively. Now we can assume, by induction on $n$, that $I(\bar{S}, \bar{T})=\left(\bar{S}^{\prime}, \bar{T}^{\prime}\right)$ has been defined. So let $I(S, T)=\left(S^{\prime}, T^{\prime}\right)$ where $S^{\prime}$ is $\bar{S}^{\prime}$ with a hook of size 1 added to the end of the first column and $T^{\prime}$ is $\bar{T}^{\prime}$ with a cell labeled $n$ added to the end of the first column. Clearly this will result in a sign-reversing involution as long as this was true for pairs with $n-1$ cells. So for the rest of this section we will also assume that the cell containing $n$ in $T$ corresponds to a cell in a hook of at least two cells in $S$.

To describe $I$ under these assumptions, we will need names for the different parts of a rim hook $H$. As usual, let $(i, j)$ denote the cell of a shape $\lambda$ in row $i$ and column $j$. An internal corner of $H$ is $(i, j) \in H$ such that $(i+1, j),(i, j+1) \in H$. Dually, an external corner of $H$ is $(i, j) \in H$ such that $(i-1, j),(i, j-1) \in H$. The head of $H$ is the $(i, j) \in H$ with smallest $i$ and largest $j$. Similarly, the tail of $H$ is the $(i, j) \in H$ with largest $i$ and $j=1$. In the previous example, the upper hook of size 4 has internal corner $(1,2)$, external corner $(2,2)$, head $(1,3)$, and tail $(2,1)$. The permissible cells of $H$ are precisely those which are either an internal corner, external corner, head, or tail.

Now define a rooted Ferrers diagram to be a Ferrers diagram where one of the nodes has been marked. We will indicate this in our figures by making the distinguished node a square. Any tableau built out of a Ferrers diagram can be rooted in an analogous way. Any invariants of the original tableau will be carried over to the rooted version without change. Now associate with any pair $(S, T)$ in the sum (1) a rooted special rim-hook tableau $\dot{S}$ by rooting $S$ at the node where the entry $n$ occurs in $T$. We will define a sign-reversing involution $\iota$ on the set of rooted special rim-hook tableaux of 
given type which are obtainable in this way. In addition, $\iota$ will have the property that if $\iota(\dot{S})=\dot{S}^{\prime}$ and $\dot{S}, \dot{S}^{\prime}$ have roots $r, r^{\prime}$ respectively, then

$$
\operatorname{sh}(\dot{S})-r=\operatorname{sh}\left(\dot{S}^{\prime}\right)-r^{\prime}
$$

where the minus sign represents set-theoretic difference of diagrams. Our full involution $I(S, T)=\left(S^{\prime}, T^{\prime}\right)$ will then be the composition

$$
(S, T) \longrightarrow \dot{S} \stackrel{\iota}{\longrightarrow} \dot{S}^{\prime} \longrightarrow\left(S^{\prime}, T^{\prime}\right)
$$

where $S^{\prime}$ is obtained from $\dot{S}^{\prime}$ by forgetting about the root and $T^{\prime}$ is obtained by replacing the root of $\dot{S}^{\prime}$ by $n$ and leaving the numbers $1,2, \ldots, n-1$ in the same positions as they were in $T$. Note that (2) guarantees that $T^{\prime}$ is well defined. Furthermore, it is clear from construction that $I$ will be a sign-reversing involution because $\iota$ is. Even though $\iota$ has not been fully defined, we can give an example of the rest of the algorithm as follows.

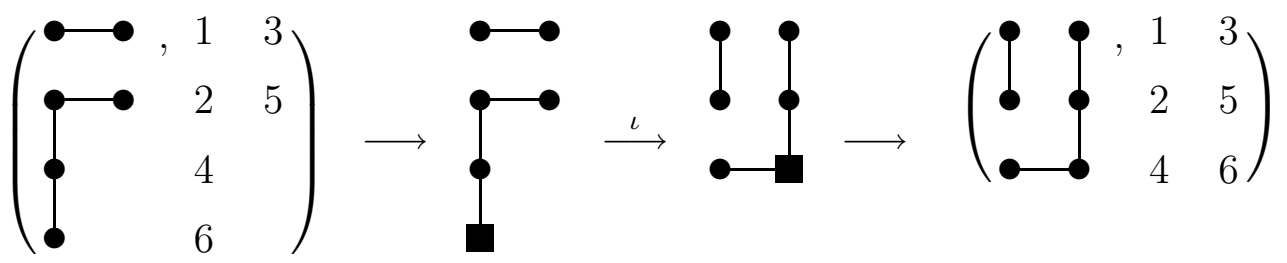

To define $\iota$ precisely, we will need to enlarge the set of tableaux under consideration. An overlapping rooted special rim-hook tableau is a way of writing a shape as a union of special rim-hooks all of which are disjoint with the exception of two whose intersection is precisely the root, and this root must be a permissible element of both hooks. Furthermore, one of the two hooks containing the root will be designated as active. In our diagrams, the active hook will have all of its nodes enlarged. In a non-overlapping rooted special rim-hook tableau, the unique hook containing the root is considered active.

We are now ready to fully describe the involution $\iota$. Starting with an appropriately rooted special rim-hook tableau $\dot{S}$, we will generate a sequence of tableaux

$$
\dot{S}=\dot{S}_{0}, \dot{S}_{1}, \dot{S}_{2}, \ldots, \dot{S}_{t}=\iota(\dot{S}) .
$$

For $0<i<t, \dot{S}_{i}$ will be an overlapping rooted special rim-hook tableau of shape $\operatorname{sh}(\dot{S})-r$ where $r$ is the root of $\dot{S}$. Finally, $\dot{S}_{t}$ will be a non-overlapping rooted special rim-hook tableau satisfying (2). All tableaux in the sequence will have the same type. There are five operations for obtaining $\dot{S}_{i}$ from $\dot{S}_{i-1}, 1 \leq i \leq t$, as follows. Whichever operation is applied, the designation of "active" is transfered from the hook that was modified to the new hook which it overlaps (except in the last step where no new hook is overlapped and so the hook which was active remains so). Throughout, $r$ is the current root.

CO If $r$ is an internal or external corner of the active hook, reflect the root in a line containing the two nodes of the hook to which it is adjacent. 
SI If the active hook of size one, i.e., consists only of $r$, then it must be at the top or bottom of the first-column portion of the other hook containing it (since all roots are at permissible nodes). Move the hook of size one and the root to the opposite end of that portion of the other hook.

HE If $r$ is at the head of the active hook of size at least two, then remove it and attach it just below the tail of this hook.

TV If $r$ is at the tail of the active hook of size at least two and the next node of the hook is directly above, then remove it and attach it to the head of the active hook. Because of permissibility, exactly one of the two ways to attach the root (vertically or horizontally) will be possible.

TH If $r$ is at the tail of the active hook of size at least two and the next node of the hook is directly to the right, note that $r$ must also be at the tail of the other hook containing it (because of permissibility) and so the hooks must have different sizes (because they are special). Let $s$ be the size of the smaller hook and let $v$ be the $(s+1)$ st node from $r$ in the bigger hook. Remove the portion of the bigger hook from $v$ on and attach it to the end of the smaller hook. The root stays in the same place.

Here is an example of the application of these rules to construct $\iota(\dot{S})$. Each step is labeled with the operation being used. When $\mathrm{TH}$ is applied, the vertex $v$ is marked.
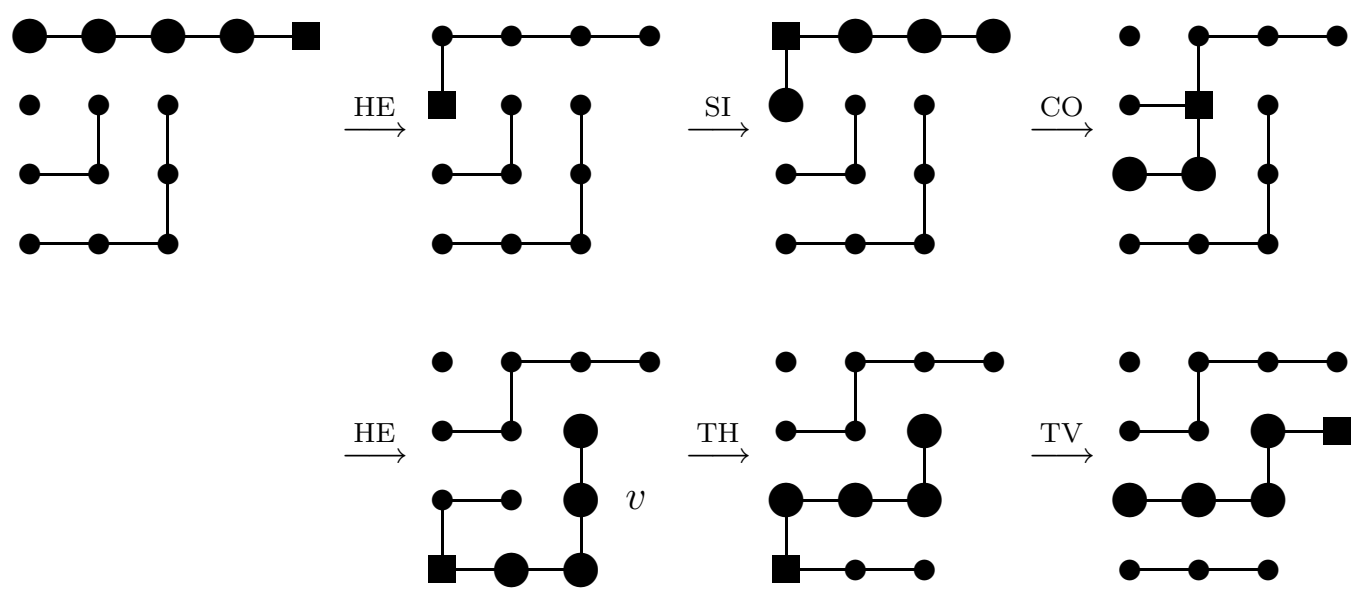

We must now show that $\iota$ is a well-defined sign-reversing involution. By examining each of the rules in turn it is easy to see that all the rim-hook tableaux generated have the same type and that the root is always in a permissible cell. Also it is clear that each rule can be reversed: $\mathrm{CO}$, SI, and TH are self-inversive while $\mathrm{HE}$ and TV are inverses of each other. So if we do a single step without changing which hook is designated as active and then apply the rules again we will return to the original tableau. This means that $\iota$ will be an involution if it is well defined.

To finish the proof of well definedness, we must show that the algorithm terminates, i.e., that eventually a non-overlapping tableau is produced. Suppose to the contrary 
that (3) goes on forever. Since there are only a finite number of tableaux of a given type, this sequence must repeat. Let $\dot{S}_{i}$ have the smallest index such that $\dot{S}_{i}=\dot{S}_{j}$ for some $j>i$. Note that since we are assuming that all tableaux other than $\dot{S}_{0}$ are overlapping, we must have $i \geq 1$. But we saw in the previous paragraph that each step of the algorithm is invertible and $i \geq 1$, so we must have $\dot{S}_{i-1}=\dot{S}_{j-1}$. This contradicts the minimality of $i$ and proves termination.

It remains to show that $\iota$ is sign reversing. In fact, we will prove the stronger statement that the sign of $\dot{S}_{i}$ depends only on the type of active hook it contains and the sign of $\dot{S}$. We will label the active hook in a tableau with the same pair of letters used for the rule which can be applied to it. We will also have to refine CO into two types of hooks, namely CI (respectively, CE) if the corner is internal (respectively, external). So in the previous example, the CO hook in the third tableau would be classified as a CI. Similarly, we have to split HE into two types: HV (respectively, HH) if the head of the hook is directly above (respectively, to the right of) its predecessor in the hook. Looking at the previous example again, the initial active hook is an $\mathrm{HH}$ while the fourth one is an HV.

Lemma 2.1. Let $H_{i}$ be the active hook in $\dot{S}_{i}$ from the sequence (3) where $0 \leq i<t$. Then

$$
\epsilon\left(\dot{S}_{i}\right)= \begin{cases}\epsilon(\dot{S}) & \text { if } H_{i} \text { is } \mathrm{CE}, \mathrm{HH}, \text { or } \mathrm{TV} \\ -\epsilon(\dot{S}) & \text { if } H_{i} \text { is } \mathrm{CI}, \mathrm{HV}, \text { or } \mathrm{TH}\end{cases}
$$

Proof. We induct on $i$. Suppose first that $i=0$ so that $\dot{S}_{0}=\dot{S}$. Since $n$ must be at the end of a row and column, the only possibilities for $H_{0}$ are $\mathrm{CE}, \mathrm{HH}$, or TV. Clearly we also have $\epsilon\left(\dot{S}_{0}\right)=\epsilon(\dot{S})$, and so the lemma is true in this case.

The induction step breaks down into six cases depending upon the nature of $H_{i}$. Since they are all similar, we will do the one for $H_{i}=\mathrm{TV}$ and then the other five can be verified by the reader if they wish.

Note first that because of the way the root moves in each of the steps, TV can only be preceded by $\mathrm{CE}, \mathrm{TH}, \mathrm{TV}$, or SI. (For example, after a CI step the root cannot be in the first column and so it can't precede TV.)

If $H_{i-1}$ is $\mathrm{CE}$ then by induction and the fact that a $\mathrm{CE}$ step does not change the number of vertical edges in a hook

$$
\epsilon\left(\dot{S}_{i}\right)=\epsilon\left(\dot{S}_{i-1}\right)=\epsilon(\dot{S})
$$

If $H_{i-1}$ is $\mathrm{TH}$ then this step changes the number of vertical edges in the overlapping hooks by \pm 1 . So by induction again

$$
\epsilon\left(\dot{S}_{i}\right)=-\epsilon\left(\dot{S}_{i-1}\right)=-(-\epsilon(\dot{S}))=\epsilon(\dot{S}) .
$$

If $H_{i-1}$ is $\mathrm{TV}$, then in order for it to overlap $H_{i}$ by a node in the first column it must be that $H_{i-1}$ consists precisely of a strip of nodes in the first column directly below the root of $H_{i}$. So the TV step does not change the number of vertical edges in $H_{i-1}$ and so the same equalities as for the $\mathrm{CE}$ case give the desired conclusion. 
Finally, consider what happens if $H_{i-1}$ is SI. Then in $\dot{S}_{i-1}$, the singleton hook must be at the top of the portion of $H_{i}$ in the first column. So $H_{i-2}$ must have been either a $\mathrm{CE}$ (if $H_{i}$ contains nodes outside of the first column) or a TV of the type discussed in the previous paragraph. In either case, the passage from $H_{i-2}$ to $H_{i-1}$ causes no change in sign and neither does the SI step. So by induction

$$
\epsilon\left(\dot{S}_{i}\right)=\epsilon\left(\dot{S}_{i-2}\right)=\epsilon(\dot{S})
$$

This completes the demonstration that the lemma holds in the TV case.

The astute reader will have noticed that this lemma did not deal with the case when $H_{i}$ is SI. But then $\epsilon\left(S_{i}\right)=\epsilon\left(S_{i+1}\right)$ where $H_{i+1}$ is one of the cases covered, so there is no harm in the omission. Now the following theorem will complete the proof that $\iota$, and hence $I$, is a well-defined, sign-reversing involution.

Theorem 2.2. If $\iota(\dot{S})=\dot{S}^{\prime}$ then $\epsilon\left(\dot{S}^{\prime}\right)=-\epsilon(\dot{S})$

Proof. We continue to use the notation in (3) where $\dot{S}^{\prime}=\dot{S}_{t}$. Since $\dot{S}_{t}$ is nonoverlapping, $H_{t}$ must be one of $\mathrm{CE}, \mathrm{HH}$, or TV.

If $H_{t}$ is $\mathrm{CE}$ then $H_{t-1}$ must have been CI. So using the previous lemma with $i=t-1$ and the fact that a CI step does not change sign gives

$$
\epsilon\left(\dot{S}^{\prime}\right)=\epsilon\left(\dot{S}_{t-1}\right)=-\epsilon(\dot{S}) .
$$

If $H_{t}$ is $\mathrm{HH}$ then $H_{t-1}$ must have been TV. Furthermore, this TV step must have added a horizontal step to $H_{t-1}$ and so decreased the number of vertical edges by one. So using the lemma again gives

$$
\epsilon\left(\dot{S}^{\prime}\right)=-\epsilon\left(\dot{S}_{t-1}\right)=-\epsilon(\dot{S}) .
$$

Finally, suppose $H_{t}$ is TV. Then $H_{t-1}$ is either HH or HV and either the previous string of equalities or the one before that, respectively, hold. So in all cases $\epsilon\left(\dot{S}^{\prime}\right)=$ $-\epsilon(\dot{S})$.

\section{The $(\mathbf{3}+\mathbf{1})$-Free ConjeCture}

In order to make a connection of our work with the $(\mathbf{3}+\mathbf{1})$-free Conjecture, we first need to introduce Stanley's chromatic symmetric function $[11,12]$. Let $G=(V, E)$ be a graph with a finite set of vertices $V$ and edges $E$. A proper coloring of $G$ from a set $A$ is a function $\kappa: V \rightarrow A$ such that $u v \in E$ implies $\kappa(u) \neq \kappa(v)$. Now consider a countably infinite set of variables $\mathbf{x}=\left\{x_{1}, x_{2}, \ldots\right\}$. Stanley associated with each graph a formal power series

$$
X_{G}=X_{G}(\mathbf{x})=\sum_{\kappa: V \rightarrow \mathbb{P}} x_{\kappa\left(v_{1}\right)} x_{\kappa\left(v_{2}\right)} \cdots x_{\kappa\left(v_{n}\right)}
$$

where $\kappa$ is a proper coloring from the positive integers $\mathbb{P}$. Note that if one sets $x_{1}=$ $x_{2}=\ldots=x_{n}=1$ and $x_{i}=0$ for $i>n$, denoted $\mathbf{x}=1^{n}$, then $X_{G}$ reduces to the number of proper colorings of $G$ from a set with $n$ elements. So under this substitution, 
$X_{G}\left(1^{n}\right)=P_{G}(n)$ where $P_{G}(n)$ is the famous chromatic polynomial of Whitney [17]. Also, because permuting the colors of a proper coloring keeps the coloring proper, $X_{G}(\mathbf{x})$ is in the algebra $\Lambda(\mathbf{x})$ of symmetric functions in $\mathbf{x}$ over the rationals. In [11, 12], Stanley was able to derive many interesting properties of the chromatic symmetric function $X_{G}(\mathbf{x})$ some of which generalize those of the chromatic polynomial and some of which cannot be interpreted after substitution.

One natural question to ask is whether one can say anything about the expansion of $X_{G}(\mathbf{x})$ in any of the usual bases for $\Lambda(\mathbf{x})$. If $f \in \Lambda(\mathbf{x})$ and $\left\{b_{\lambda}: \lambda \in \operatorname{Par}\right\}$ is a basis for $\Lambda(\mathbf{x})$, then we will say that $f$ is b-positive if in the expansion $f=\sum_{\lambda} c_{\lambda} b_{\lambda}$, all of the coefficients satisfy $c_{\lambda} \geq 0$. The $(\mathbf{3}+\mathbf{1})$-free Conjecture states that for certain graphs, $X_{G}$ is $e$-positive where the $e_{\lambda}$ are the elementary symmetric functions.

To describe the appropriate graphs for the conjecture, consider a finite poset (partially ordered set) $(P, \leq)$. We say that $P$ is $(\mathbf{a}+\mathbf{b})$-free if it contains no induced subposet isomorphic to a disjoint union of an $a$-element chain and a $b$-element chain. Also, given any poset $P$, we can form its incomparability graph, $G(P)$, having vertices $V=P$ and an edge between $u$ and $v$ in $G(P)$ if and only if $u$ and $v$ are incomparable in $P$. Through their work on immanants of Jacobi-Trudi matrices, Stanley and Stembridge [14] were led to the following conjecture.

Conjecture 3.1 ((3+1)-free Conjecture). Let $P$ be a a $(\mathbf{3}+\mathbf{1})$-free poset. Then $X_{G(P)}$ is e-positive, i.e., if

$$
X_{G(P)}=\sum_{\mu} c_{\mu} e_{\mu}
$$

then $c_{\mu} \geq 0$ for all $\mu$.

There is a fair amount of evidence to support this conjecture. Stembridge has verified that it is true for all $884(\mathbf{3}+\mathbf{1})$-free posets having at most 7 elements. Gebhard and Sagan [4] have used the theory of symmetric functions in noncommuting variables to prove that the conjecture holds for certain posets which are both $(\mathbf{3}+\mathbf{1})-$ and $(\mathbf{2}+\mathbf{2})$ free.

One of the most significant results about this conjecture was obtained by Gasharov [5] who proved that if $P$ is $(\mathbf{3}+\mathbf{1})$-free then $X_{G(P)}$ is $s$-positive where $s_{\lambda}$ is the Schur function corresponding to $\lambda$. He did this by giving a combinatorial interpretation to the coefficients in the $s$-expansion of $X_{G(P)}$ which we will need in the sequel.

For a poset $P$, a $P$-tableau $T$ of shape $\lambda$ is a filling of the cells of $\lambda$ with the elements of $P$ (each used exactly once) such that for all $(i, j) \in \lambda$ :

(1) $T_{i, j}<T_{i+1, j}$, and

(2) $T_{i, j} \ngtr T_{i, j+1}$

where a condition is considered vacuously true if subscripts refer to a cell outside of $\lambda$. Note that when $P$ is a chain, then a $P$-tableau is just a standard Young tableau. Letting $f_{P}^{\lambda}$ denote the number of $P$-tableaux of shape $\lambda$, Gasharov proved the following result. 
Theorem 3.2 (Gasharov). If $P$ is $(\mathbf{3}+\mathbf{1})$-free then

$$
X_{G(P)}=\sum_{\lambda} f_{P}^{\lambda} s_{\lambda^{\prime}}
$$

where $\lambda^{\prime}$ is the conjugate of $\lambda$.

Note that this immediately implies $s$-positivity.

Chow [1] pointed out that (5) could be combined with Eğecioğlu and Remmel's result to obtain a combinatorial interpretation of the coefficients $c_{\mu}$ in (4). First note that the change of basis matrix between the Schur and elementary symmetric functions is

$$
s_{\lambda^{\prime}}=\sum_{\mu} K_{\mu, \lambda}^{-1} e_{\mu}
$$

Combining this with (5) we get

$$
X_{G(P)}=\sum_{\lambda, \mu} K_{\mu, \lambda}^{-1} f_{P}^{\lambda} e_{\mu}
$$

Since the $e_{\mu}$ are a basis, we have

$$
c_{\mu}=\sum_{\lambda} K_{\mu, \lambda}^{-1} f_{P}^{\lambda}
$$

Finally we apply Theorem 1.1 to get the desired interpretation.

Corollary 3.3 (Chow). The coefficients $c_{\mu}$ in the e-expansion of $X_{G}(P)$ satisfy

$$
c_{\mu}=\sum_{(S, T)} \epsilon(S)
$$

where the sum is over all pairs of a special rim hook tableau $S$ of type $\mu$ and a P-tableau $T$ with the same shape as $S$.

Note that a column of a $P$-tableau $T$ must be a chain in $P$ and the number of rim hooks in $S$ is at most the length of its first column because they are special. So the previous corollary implies that $c_{\mu}=0$ whenever $\mu$ has more parts than the height of $P, h(P)$ (which is defined as the number of elements in the longest chain of $P$ ). So this cuts down on the number of coefficients which we need to consider.

So to show that $c_{\mu} \geq 0$, it suffices to find an involution $I$ on the pairs in the previous corollary such that if $I(S, T)=\left(S^{\prime}, T^{\prime}\right)$ then

(1) $(S, T)=\left(S^{\prime}, T^{\prime}\right)$ implies $\epsilon(S)=1$, and

(2) $(S, T) \neq\left(S^{\prime}, T^{\prime}\right)$ implies $\epsilon\left(S^{\prime}\right)=-\epsilon(S)$.

The involution in the previous section does this when $P$ is a chain (so that $P$-tableaux are standard Young tableaux). Of course, then $G(P)$ is a totally disconnected graph and thus $X_{G(P)}=e_{\left(1^{n}\right)}$ directly from the definition of $X_{G}$. So it would be nice to apply these ideas to a less obvious case. This will be done in the next theorem which was also derived by Stanley and Stembridge themselves [14] using the theory of rook 
placements. Note that if $h(P) \leq 2$ then $P$ must be $(\mathbf{3}+\mathbf{1})$-free since it does not even contain a 3 -element chain.

Theorem 3.4 (Stanley-Stembridge). If $h(P) \leq 2$ then $X_{G(P)}$ is e-positive.

Proof. It suffices to construct the involution $I$ in this case. First of all, the remarks after Corollary 3.3 show that any $P$-tableau, $T$, will have at most two rows since $h(P) \leq 2$. If a special rim-hook tableau $S$ has at most two rows and $\epsilon(S)=-1$, then it must have exactly two rows and contain exactly one vertical edge.

So let $(S, T)$ be a pair having common shape $\lambda=\left(\lambda_{1}, \lambda_{2}\right)$ and with $\epsilon(S)=-1$. Let $\dot{S}$ be the tableau obtained by rooting $S$ at the end of the second row. Note that a priori it is not clear that this is the best place to root $S$ since $P$ may have more than one maximal element. But we will see that this choice will work well.

Now form $\dot{S}^{\prime}=\iota(\dot{S})$. By our assumptions on $S, \iota$ will consist of a sequence of steps resulting in an $\dot{S}^{\prime}$ which will be the unique tableau of shape $\left(\lambda_{1}+1, \lambda_{2}-1\right)$ where each row is a special rim hook and the root is at the end of the first row. We now obtain $\left(S^{\prime}, T^{\prime}\right)=I(S, T)$ as before by removing the root from $\dot{S}^{\prime}$ to get $S^{\prime}$ and getting $T^{\prime}$ by leaving all the elements of $T$ in the same place except the one, $x$, which was at the end of the second row in $T$ and is now at the end of the first row in $T^{\prime}$. Clearly this reverses sign, but we must check that $T^{\prime}$ is still a valid $P$-tableau. But $x$ was at the end of a column of length two in $T$ and so cannot have any element above it in $P$ which is of height two. So the row condition for $P$-tableaux will still be satisfied and $I$ is well defined.

In order to turn $I$ into an involution, we must characterize those pairs $\left(S^{\prime}, T^{\prime}\right)$ which are in the image of the function so far and map them back to their preimages. So suppose the tableaux in $\left(S^{\prime}, T^{\prime}\right)$ have shape $\nu=\left(\nu_{1}, \nu_{2}\right)$ where $\nu_{1}>\nu_{2}+1>0$ and $\epsilon\left(S^{\prime}\right)=+1$. Consider the elements $x=T_{1, \nu_{1}}^{\prime}$ and $y=T_{1, \nu_{2}+1}^{\prime}$. Then it is easy to see that the image of $I$ is precisely the set of all such pairs such that $x>y$ in $P$. So we can reverse the algorithm by forming $\dot{S}^{\prime}$ which is $\dot{S}$ rooted at the end of the first row, applying $\iota$, and then using the usual operations to recover $(S, T)$.

Finally, we need to consider what happens to the $(S, T)$ which have not been paired up so far by $I$. But since these all have positive sign, we can just make them fixed points of $I$. This completes the definition of $I$ and the proof of the Theorem.

As an example of the algorithm in the previous proof, consider the poset

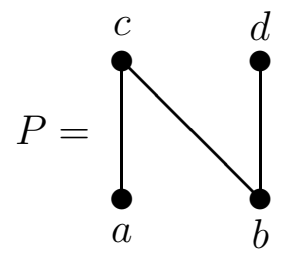


There are 20 pairs $(S, T)$ for this poset. To present them in an economical way, we will combine each pair into a single tableau with elements in the same places as in $T$ and edges between pairs of elements which are adjacent in a hook of $S$. With this notation, tableaux which are matched by $I$ are as follows.

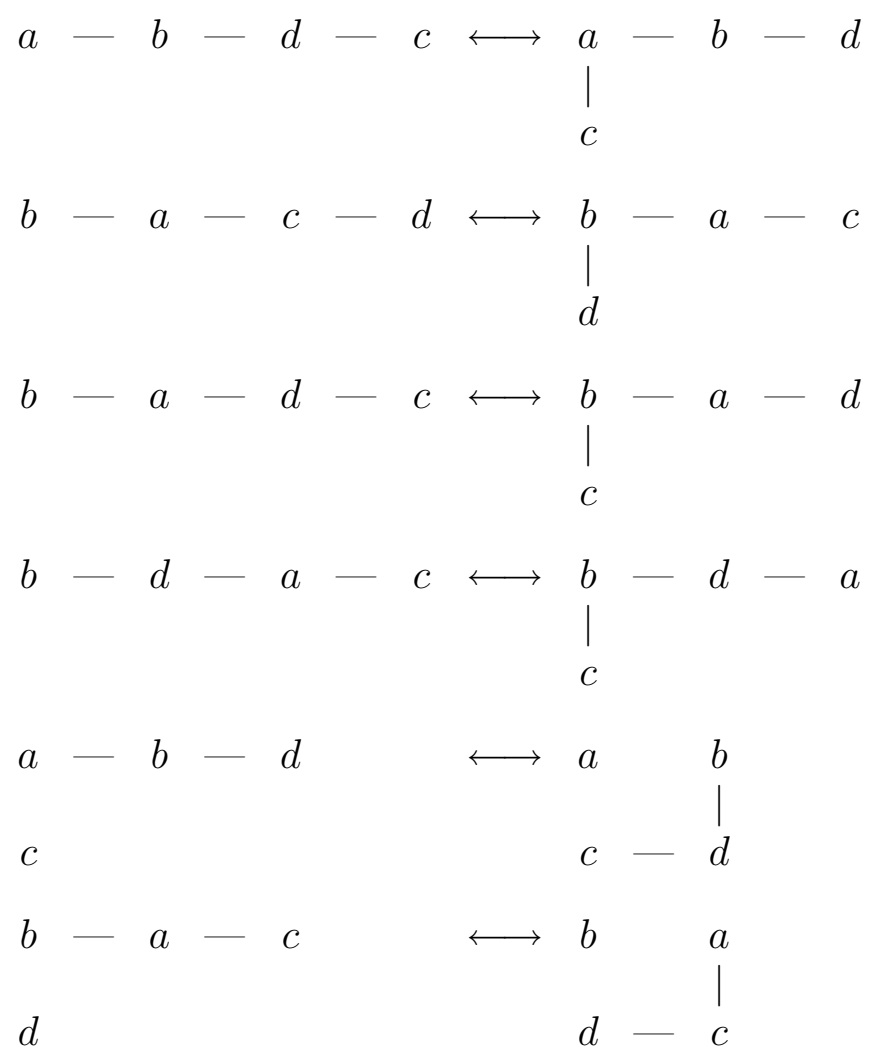

And here is a list of the fixed points organized in columns by shape.

$$
\begin{array}{ccc}
a-b-c-d & b-a-d & a-b \\
c & b-d-a & b-a \\
b-c-d-a & c & d-c \\
c-d-a-b & \\
d-a-b-c &
\end{array}
$$

Counting the fixed points by shape, we immediately have

$$
X_{G(P)}=4 e_{(4)}+2 e_{(3,1)}+2 e_{(2,2)} .
$$


We remark that if one adjoins a unique maximum or minimum to $P$, then this adds

an isolated vertex to $G(P)$. So this just multiplies $X_{G(P)}$ by $e_{(1)}$. So the previous theorem implies that $X_{G(P)}$ is e-positive in the case when $h(P)=3$ and $P$ has a unique maximum or minimum as well as in the case when $h(P)=4$ and $P$ has both.

\section{FURTHER WORK}

We hope that the involution we have presented will just be a first step towards making progress on the problem of Ĕgecioğlu and Remmel as well as on the $(\mathbf{3}+\mathbf{1})$-free Conjecture. In order to encourage the reader to develop these ideas, let us present some thoughts about how to proceed.

To complete a combinatorial proof of $K^{-1} K=I$, we must find an involution $I$ on pairs $(S, T)$ of the same shape for any given type $t(S)=\mu$ and content $c(T)=\nu$. Of course, $I$ should be sign reversing on all its 2-cycles and any fixed points should have $S$ of positive sign. If $\mu \neq \nu$ then there should be no fixed points. If $\mu=\nu$ then there should be a single fixed point which should probably be the unique pair of shape $\mu=\nu$.

If the largest element of $T$ to appear in a hook of size at least two only occurs once in $T$, then one can use the same algorithm as before to construct $I$. The question is what to do if the largest element of $T$ occurs with nontrivial multiplicity. One possible solution is to recall that to every semistandard $T$ there is a canonically associated standard Young tableau $T_{0}$ obtained by labeling the 1's in $T$ from left to right with $1,2, \ldots, \nu_{1}$, then labeling the 2 's in the same manner with $\nu_{1}+1, \nu_{1}+2, \ldots, \nu_{1}+\nu_{2}$, and so forth. One can now apply the old involution $I$ to the pair $\left(S, T_{0}\right)$ to obtain a pair $\left(S^{\prime}, T_{0}^{\prime}\right)$. The problem is that if we now reverse the standardization procedure, we may no longer get a semistandard tableau as one may get two largest elements in the same column. But perhaps there is a way to circumvent this.

Another possible approach would be to try and come up with a new set of rules where all the largest elements were moved as a group in each step. We have been able to see how some pairs might behave under this assumption, but have not been able to come up with something that works in all circumstances.

The difficulties when dealing with posets are similar. Since $P$ may have many maximal elements, it is unclear which of them should be used to root $S$. Or maybe it is the case that the root can be chosen arbitrarily among these maximals as long as one always chooses the same element. But some extra idea will have to be incorporated to deal with the fact that a maximal element need not be at the end of a row (although it must be at the end of a column) and to make sure that when the maximal element is moved to the new position, the new array remains a $P$-tableau. This should be where the $(\mathbf{3}+\mathbf{1})$-free condition comes in.

\section{REFERENCES}

[1] T. Chow, A note on a combinatorial interpretation of the e-coefficients of the chromatic symmetric function, preprint (1997), 9 pp, arXiv math.CO/9712230. 
[2] Ö. Eğecioğlu and J. Remmel, A combinatorial interpretation of the inverse Kostka matrix, Linear and Multilinear Algebra 26 (1990), 59-84.

[3] W. Fulton, "Young Tableaux," London Mathematical Society Student Texts 35, Cambridge University Press, Cambridge, 1999.

[4] D. Gebhard and B. Sagan, A chromatic symmetric function in noncommuting variables, J. Algebraic Combin. 13 (2001), 227-255.

[5] V. Gasharov, Incomparability graphs of $(\mathbf{3}+\mathbf{1})$-free posets are $s$-positive, Discrete Math $\mathbf{1 5 7}$ (1996), 193-197.

[6] I. Gessel and G. Viennot, Binomial determinants, paths, and hook length formulae, Adv. in Math. 58 (1985), 300-321.

[7] I. Gessel and G. Viennot, Determinants, paths, and plane partitions, in preparation.

[8] B. Lindström, On the vector representation of induced matroids, Bull. London Math. Soc. 5 (1973), 85-90.

[9] I. G. Macdonald, "Symmetric functions and Hall polynomials," 2nd edition, Oxford University Press, Oxford, 1995.

[10] B. Sagan, "The Symmetric Group: Representations, Combinatorial Algorithms, and Symmetric Functions," 2nd edition, Springer-Verlag, New York, 2001.

[11] R. P. Stanley, A symmetric function generalization of the chromatic polynomial of a graph, Advances in Math. 111 (1995), 166-194.

[12] R. P. Stanley, Graph Colorings and related symmetric functions: ideas and applications: A description of results, interesting applications, \& notable open problems, Selected papers in honor of Adriano Garsia (Taormina, 1994) Discrete Math. 193 (1998), 267-286.

[13] R. P. Stanley, "Enumerative Combinatorics, Volume 2," Cambridge University Press, Cambridge, 1999.

[14] R. P. Stanley and J. Stembridge, On immanants of Jacobi-Trudi matrices and permutations with restricted position, J. Combin. Theory Ser. A 62 (1993), 261-279.

[15] D. Stanton and D. White, A Schensted correspondence for rim hook tableaux, J. Combin. Theory Ser. A 40 (1985), 211-247.

[16] D. White, A bijection proving orthogonality of the characters of $S_{n}$, Adv. in Math. 50 (1983), 160-186.

[17] H. Whitney, A logical expansion in mathematics, Bull. Amer. Math. Soc. 38 (1932), 572-579.

Department of Mathematics, Michigan State University, East Lansing, Mi 488241027, USA

E-mail address: sagan@math.msu.edu

Department of Mathematics, Hallym University, Chunchon, KOREA 200-702

E-mail address: jjlee@hallym.ac.kr 